\title{
Performance Evaluation of Zone-Based Routing with Hierarchical Routing in Wireless Sensor Networks
}

\author{
Rab Nawaz Jadoon (iD, ${ }^{1,2}$ WuYang Zhou $\mathbb{D}^{1},{ }^{1}$ Iftikhar Ahmed Khan $\mathbb{D}^{2}{ }^{2}$ \\ Muhammad Amir Khan $\mathbb{D}^{3},{ }^{3}$ Shahbaz Akhtar Abid, ${ }^{4}$ and Nauman Ali Khan $\mathbb{D}^{1}$ \\ ${ }^{1}$ School of Information Science and Technology, University of Science and Technology of China, Hefei 230000, China \\ ${ }^{2}$ Department of Computer Science, COMSATS University, Islamabad-Abbottabad Campus 22060, Abbottabad, Pakistan \\ ${ }^{3}$ Department of Electrical Engineering, COMSATS University, Islamabad-Abbottabad Campus 22060, Abbottabad, Pakistan \\ ${ }^{4}$ Department of Computer Science, COMSATS University Islamabad, Lahore Campus 540000, Lahore, Pakistan
}

Correspondence should be addressed to Rab Nawaz Jadoon; rabnawaz@mail.ustc.edu.cn and WuYangZhou; wyzhou@ustc.edu.cn

Received 15 July 2019; Revised 5 September 2019; Accepted 17 September 2019; Published 13 October 2019

Guest Editor: Dionisis Kandris

Copyright (C) 2019 Rab Nawaz Jadoon et al. This is an open access article distributed under the Creative Commons Attribution License, which permits unrestricted use, distribution, and reproduction in any medium, provided the original work is properly cited.

\begin{abstract}
Routing remains a most challenging task in sensor networks because of constrained resources like battery power, processing, and memory. Many energy efficiency techniques for the sensor networks have been proposed, among which hierarchical routing is considered the most energy-efficient and extended network lifetime technique. This technique has a lesser number of transmissions in the network. On the contrary, zone-based routing claims lesser control and routing overhead on the overall network lifetime. In this research, a simulation-based comparison of zone-based routing with static clustering hierarchical routing is conducted. The simulation results show that the zone-based routing outperforms hierarchical routing with static clustering in terms of energy efficiency, network lifetime, and throughput.
\end{abstract}

\section{Introduction}

Wireless sensor networks (WSNs) have spatially distributed nodes used to sense the physical phenomenon of interest. The nodes in the networks are wirelessly connected with each other [1-4]. Each network has one or more base stations (BSs) used to gather the data from the sensors. Nodes in the network have the capability to sense, process, and route the data efficiently to the desired destination. The main issue associated with these networks is that they are constrained by energy, processing power, bandwidth, and internal memory $[5,6]$. It is highly environment and application specific that once the nodes are deployed, it is almost impossible to reach the nodes in the deployed region to replace or recharge their battery [7]. The idea of these networks was first coined for military applications. Recently, it is applied in many different application areas spanning from environment sensing to health, engineering, agricultural, industrial, and biohabitat monitoring where sensor networks are playing a vital role [8].

Every protocol developed in the sensor networks stresses on energy efficiency to prolong the network lifetime [9]. The protocol developed at the medium access control (MAC) layer, network layer, transport layer, or the layers above these layers must be energy efficient along with possessing other QoS parameters. Routing is considered to be the most energy-intensive task in the sensor networks $[2,8]$. Various energy-efficient protocols have been developed for routing the data from the source to the destination (i.e., BS). As far as network lifetime is concerned, the sensor should consume lesser energy to route the data in the network.

For the routing purpose, the main categories are direct communication protocols, flat (multihop) routing protocols, and hierarchical routing protocols $[2,10]$. Hierarchical routing protocols are also called cluster-based protocols. Researchers have extended the developments in clusterbased routing because of the claims that it performs best because of lower transmissions to the BS as compared to other categories of the protocols.

The main drawback of direct communication protocols is that the nodes are located far away from the BS. This results in quick depletion of their energy as they need high 
energy to transmit the data to reach the ultimate destination. Direct communication is also not suitable for scalable environments because a lot of collisions happen in the system which drastically affects the network throughput.

In contrast, multihop routing seems to be realistic in the sense that whenever a node has data to send, it may reach the BS by utilizing several hops along the path. The routing strategy ensures the nodes with most energy in the path to route the data to the BS [2]. Multihop routing is best suited for the environment where node density is high and nodes are not directly reachable to the BS.

In cluster-based routing/hierarchical routing protocols, the whole sensing region is divided into different equal or unequal regions with one designed cluster head $(\mathrm{CH})$ in each region [11]. All the associated cluster nodes will send their data to the $\mathrm{CH}$ in their allotted time slot communicated beforehand, and then the $\mathrm{CH}$ performs data aggregation to send the accumulated information to the BS either directly or from the $\mathrm{CH}$ to the $\mathrm{CH}$ to the BS. The clusters are either static or dynamic. In static clustering, once the clusters are formed, they will never be changed till the end of the network. On the contrary, after each or certain round of communications, the clusters and $\mathrm{CHs}$ are rotated for balancing energy dissipation across the network [12]. In cluster-based routing, a smaller number of transmissions are carried out as only the aggregated information is transmitted to the BS. Either the clusters are formed by the BS or they organize themselves in a distributed way into a different cluster. Once we limit the transmissions in the network, ultimately, energy is saved and the overall network lifetime is extended [2,9].

In zone-based routing, the sensing region is divided into different zones as done in clustering. The difference is that it utilizes only those nodes in the network that are energy efficient. It removes the concept of the $\mathrm{CH}$ selection and its rotation after each round of communication [2]. Then, the BS is responsible for cluster formation at the start of the network. Control traffic is exchanged as well as the $\mathrm{CH}$ while setting up clusters after each round of communication in hierarchical routing. This unnecessary traffic greatly affects the overall network lifetime. If this control overhead could be eliminated, then there is a chance of network lifetime improvement.

The main motivation behind this work is to investigate and to compare the performance of zone-based routing with that of hierarchical routing, i.e., cluster-based routing. In this paper, we compare the routing algorithm using the ringzone (RARZ) [2] protocol and energy-efficient technique for handling redundant traffic (EEHRT) [9] which is purely a zone-based protocol with two well-renowned hierarchical routing protocols like the energy-efficient protocol using static clustering (EEPSC) [7] and low-energy adaptive clustering hierarchy (LEACH) $[11,13]$. The EEPSC, EEHRT, RARZ, and LEACH protocols all have the same designed philosophy of splitting the network into different zones through the BS. But the routing strategy used in them is different.
In this paper, we consider the same network model as used in the RARZ [2], EEHRT [9], EEPSC [7], and LEACH [11], with the following assumptions:

(i) All the nodes in the network are immobile

(ii) The BS is responsible for dividing the region into different clusters/zones

(iii) The nodes are homogeneous with limited battery power

(iv) BS location can be set up either inside or outside the sensing region

(v) The data sampling rate is fixed

The brief overview of the description of all the protocols presented in this paper is under Section 3. Following are some points that distinguish the RARZ routing from EEPSC routing:

(i) The RARZ and EEHRT did not utilize the concept of $\mathrm{CH}$ selection and $\mathrm{CH}$ to $\mathrm{CH}$ or $\mathrm{CH}$ to $\mathrm{BS}$ routing as done in the EEPSC and in LEACH.

(ii) The RARZ and EEHRT utilize multihop communication instead of direct communication as in the EEPSC and in the LEACH.

(iii) The RARZ and EEHRT eliminated the concept of control traffic during the routing phase while selecting the next hop node.

(iv) In the RARZ and EEHRT, the next hop node is selected on the fly without having any topological information.

(v) The RARZ and EEHRT are totally nonreactive and nonposition based.

(vi) The RARZ and EEHRT algorithms are not energy aware, but EEPSC and LEACH algorithms are energy-aware protocols. The BS knows the energy level of each node before the start of each round, and each node in the network shares its energy level to the BS.

(vii) In RARZ and EEHRT routing, the next hop node is selected based on a timer, which is a function of node residual energy and its zone where it is located.

(viii) In the EEPSC and LEACH, all the nodes in the network are assumed to be directly reachable to each other and the BS; if they are reachable to each other, then there is no need for routing. This is not a valid assumption made in the EEPSC.

After each round of communication, the $\mathrm{CHs}$ are reselected and the direct communication paradigm is adapted to route the aggregated data to the $\mathrm{BS}$ by $\mathrm{CH}$ in both the protocols (EEPSC and LEACH).

The rest of this paper is organized as follows: Section 2 describes the related work. Section 3 presents the short description of the protocols used in this paper for comparison. Section 4 describes the simulation results. Finally, Section 5 concludes this paper. 


\section{Related Works}

A lot of work regarding energy-efficient routing has been investigated in sensor networks since 2002. A plethora of work presented on routing stuff is available in the digital library. Among all the routing protocols developed till now, people have contributed a lot to cluster-based routing. The first idea of clustering was given by Wendy Heinzelman at MIT in her PhD dissertation [14], in which she developed a protocol called low-energy adaptive clustering hierarchy (LEACH) [11] which is based on distributed as well as centralized $\mathrm{CH}$ selection and cluster formation, where sensor nodes elect themselves as $\mathrm{CH}$ nodes with some probability based on remaining energy and location information. A centralized approach is also developed to form the clusters through the BS. LEACH-C [10] is based on LEACH and uses a centralized approach for cluster formation and $\mathrm{CH}$ selection. Direct communication is formulated between $\mathrm{CH}$ s and the BS. LEACH works in different phases or rounds. Each round starts with a configuration phase also called the network setup phase followed by a data communication phase. Once all the nodes associate themselves with their designated cluster, they will send their data in their allotted time slot to the $\mathrm{CH}$, and then the $\mathrm{CH}$ will send the whole cluster data after making some necessary aggregation to the BS.

Another similar solution based on hierarchical routing is presented in [5], in which each node can send its data to its immediate closest neighbour. After that, among neighbours, one leader node is selected to route the aggregated data to the BS directly. This solution was named "power-efficient gathering in sensor information systems" (PEGASIS). The main purpose of this algorithm is to evenly distribute the energy dissipation across the network.

Researchers also considered optimization of the routing process to some extent by combining different features in one algorithm. According to this design philosophy, a similar effort is done in [15], where the authors combine the data aggregation, energy-aware routing, and clustering into one protocol. This greatly enhances the overall network lifetime. The authors utilize dynamic clustering for energy balancing across the network. To conserve energy, multihop hierarchical routing is used to cover the large distances efficiently.

Another concept of energy-efficient and context-aware cluster-based routing is presented in [16] that also claims the energy-efficient communication in the system by utilizing only those nodes that are energy efficient and best suited as per the context. In ordinary clustering, the node deployment or arrangement is fixed [13,14], but people also gave a solution to implement the hierarchical routing in ad hoc way [17].

A connected cluster architecture idea is presented and tested in [16]. As per that idea, the $\mathrm{CH}$ and gateways assume the same nodes in the network. The $\mathrm{CH}$ is assumed to be the central node, while the gateway node is the backbone node to transmit data to different users placed at different locations. A distributed clustering solution is presented in [3]. According to this algorithm, nodes in the sensing region are organized into equal-sized clusters first, and then $\mathrm{CH}$ to $\mathrm{CH}$ routing is performed to extend the overall network lifetime. The energy consumption among all the CHs is evenly distributed for enhancing the network lifetime.

As sensor nodes are equipped with a built-in battery power, their lifespan is short. The researcher gave keen attention to this issue in almost every protocol developed at every layer of the protocol stack. To further enhance the network lifetime and conserve energy, a data query dissemination and gathering scheme is presented in [18]. In this work, authors conceived a concept of the parameterized query based on the user's profile to get the required data from the sensor nodes. The scheme is proven to be the most energy efficient as compared to the rest of the techniques of a similar domain.

A lot of work has also been investigated on energy-aware routing. A similar work is presented in [19], in which authors utilize an energy-aware technique with static clustering called centralized control clustering (EACCC) in order to achieve energy efficiency and greater lifespan of the network, especially in a scalable environment. The performance of the EACCC is accessed through extensive analytical proofs and simulation, and it has been shown that the EACCC is highly efficient in terms of balancing the energy consumption and prolonging the network lifetime.

The work in [17] is based on hybrid clustering. According to this scheme, clusters are static and never changed up to 10 rounds. The BS is responsible for the selection of next-phase $\mathrm{CHs}$. If the round number is less than 10, the current $\mathrm{CH}$ selects the new utmost energy level node as a $\mathrm{CH}$ and intimates its status to the BS. After round number 10, all the nodes send their energy status and location information to the BS and the BS will set up new clusters for the next time.

The researchers also presented the solutions to reduce the control overhead of the cluster-based routing protocols and to increase the network lifetime. These categories of protocols are called zone-based routing protocols [2, 9]. A similar sort of solution is presented in [3] called the zonebased routing protocol (ZBRP), which is an energy-efficient and edge-based network partitioning technique. It divides the whole sensing region into equal-sized clusters. The BS is responsible for dividing the whole sensing region into different zones around the BS, and then it further divides each zone into equal-sized clusters. Multihop cluster-based routing is performed at the start of each round of communication. New $\mathrm{CH}$ s are selected for evenly distributing the energy consumption among all the clusters and $\mathrm{CHs}$ in the network. This greatly increases the overall network lifetime as compared to the previous solutions presented in static as well as dynamic clustering. People have contributed to the area where the protocols are energy efficient along with the security measures. A similar sort of work is done in $[20,21]$. Even in the current time, the environment sensing through IoTs and other application-specific sensors are used for integration with many online forensic and real-time surveillance applications where innovative techniques are highly desirable to overcome the cybercrime issues [22]. To make the network fully connected to handle the converge 
[23] issues due to many obstacles is also highly desirable in the protocol along with other core issues like network lifetime by ensuring energy efficiency in the network.

From the literature review above, it can be concluded that researchers are striving to make protocols energy efficient as well as adding other lifetime-increasing capabilities with less control overhead. This research work also has dug another new way of designing energy-efficient routing protocols.

\section{Zone-Based Routing (ZBR) vs Hierarchical Routing (HR)}

Both zone-based routing and cluster-based routing are used interchangeably, but their routing philosophy is different. Zone-based routing claims that, along with other methods that greatly ensure energy efficiency, it has less control overhead in the network.

In this section, we present the normal working of the ring zone-based routing protocol and energy-efficient protocol using static clustering.

3.1. RARZ Routing. The ring zone-based routing protocol [2] works in the following two phases:

(a) Network configuration phase

(b) Data communication phase

3.1.1. Network Configuration Phase. This phase is also called the network setup phase in which the whole sensing region is divided into equal-sized zones. The BS is responsible for dividing the whole sensing region into equal-sized zones by sending different transmission power messages having zoneID to the sensing region. The nodes that sense that packet will set their ID as per ID received in the message. The BS broadcasts different communication range messages progressively till covering the whole field. Nodes that receive a zone $i$ message will set themselves to zone $i$, unless they have already joined the lower zoneID. All the nodes in a zone share the same zoneID. Once they set their zoneID, the zoneID will never be changed till the end of the network.

Upon successful completion of the network configuration phase, the data communication phase begins. The working phases of the RARZ are presented in Figure 1. Only the lower zone nodes will route the data of higher zones to the BS. The nodes located in the same and the higher zone will delete the packet after checking zoneID at the MAC layer. For medium access, distributed coordination function of 802.11 is used with two assumptions: (1) there is no pre-MAC addressing used and (2) it does not use strict addressing per node for communication. In RARZ routing, the BS is located among the sensor nodes, but it can be set up everywhere in the sensing region either inside or outside. BS location does not affect the data communication or routing process. just broadcasts its data with its zoneID. The nodes that hear that packet in the same, lower, and high zones will process the packet. As per the routing strategy defined in the RARZ, only the lower zone will schedule the packet further, and the same and the higher zone node will delete the packet. The nodes in the lower zone will schedule the packet for further relaying it. All the nodes in the lower zone will schedule the packet according to a timer. A timer is a function of node residual energy and its zoneID:

$$
\operatorname{timer}(t)=\alpha * z \text { one ID }+\beta\left[\frac{\mathrm{IE}}{\mathrm{CE}}\right]
$$

where zoneID is the network address of the node which is the same as that of all the nodes in a specific zone, IE is the initial energy, and CE is the current energy. The lower the ZoneID and the higher the CE, the smaller the timer, and vice versa. For the node having higher energy and lower zoneID, its timer will be expired first. $\alpha$ and $\beta$ are the weighted tuning parameters for node network address and residual energy. The values of $\alpha$ and $\beta$ are set in Table 1 . The node whose timer expires first will become the next potential hop node for the received packet. Once this node further relays the packet, the nodes in the vicinity of sensing the same packet sequence number will kill their timer and delete the packet. This protocol works efficiently without considering any location and topological information to route the packet to its ultimate destination. At each hop falling in the routing path, the most energy level nodes are selected for data transmission. The location-based routing is performed without the need for the nodes to know the position of the neighbouring node. No prior control information is needed for the next hop selection or path construction before the start of transmission. This protocol is totally blind and does routing decisions on the fly. The detailed description of the protocol is presented in [2]. In ZBR, redundant traffic is normally observed which reduces the overall network lifetime. Redundant traffic is handled in [9], to make the routing more effective w.r.t. energy efficiency and overall network lifetime.

\subsection{Energy-Efficient Technique for Handling Redundant} Traffic (EEHRT). This protocol is an extended version of the RARZ routing which handles the redundant traffic generated in zone-based routing. Moreover, the source node is acknowledged by the next hop node using a wireless broadcast advantage (WBA) technique [24] without having any special ACK packet to the sender, which ensures the reliability without incurring any extra control overhead at each hop along the routing path. The EEHRT improves the routing against the RARZ by ensuring only one copy of the packet is propagated to each hop along the routing path till reaching the BS by introducing a short beacon message in RARZ routing. The detailed working of the EEHRT is presented in [9].

\subsection{Energy-Efficient Protocol Using Static Clustering (EEPSC).} This EEPSC [7] also works in two phases: the network configuration phase and the data communication phase. 


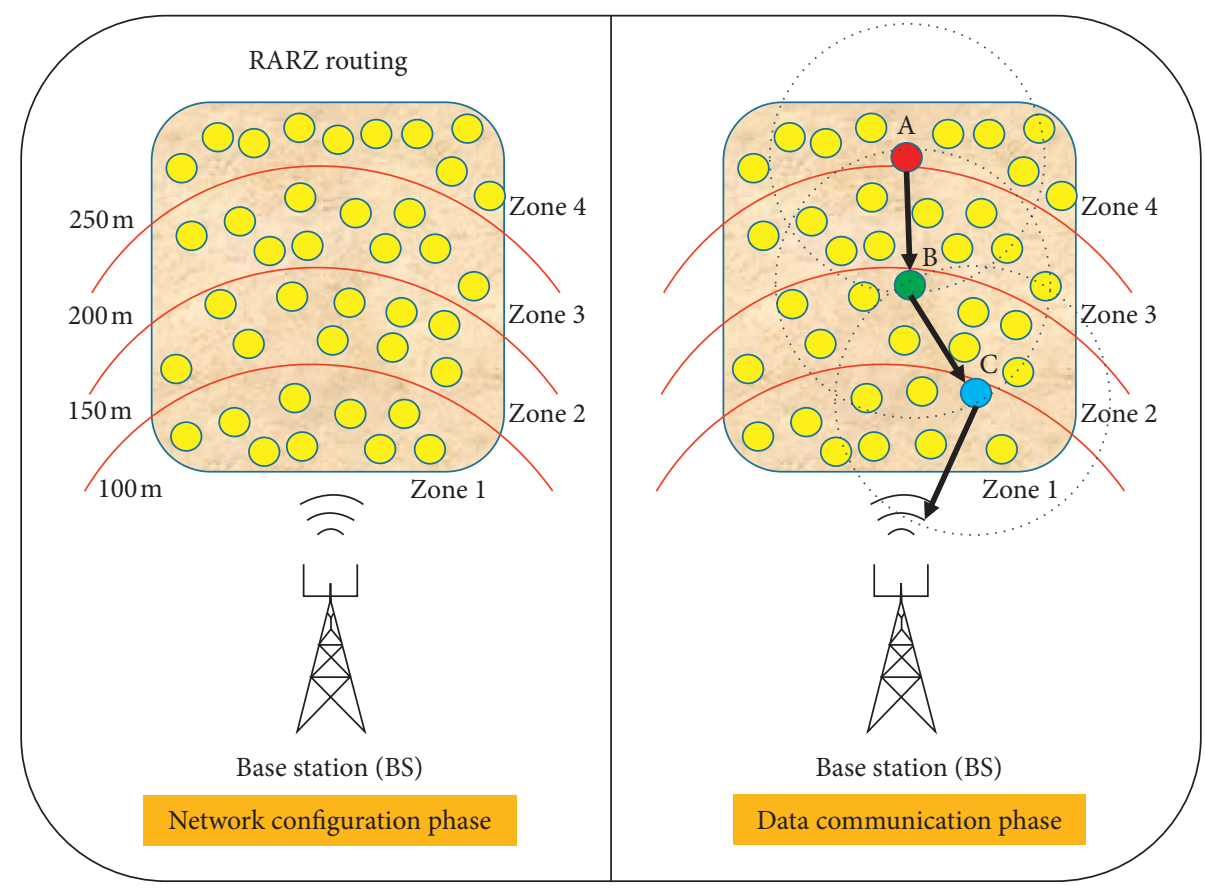

FIGURE 1: RARZ protocol phases.

TABle 1: Simulation setup.

\begin{tabular}{|c|c|c|}
\hline Type & Parameter & Value \\
\hline Network & $\begin{array}{c}\text { Size } \\
\text { Energy of each node } \\
\text { Network deployment } \\
\text { Number of zones } \\
\text { Total number of nodes }\end{array}$ & $\begin{array}{c}600 \times 600 \\
3000 \mathrm{~mJ} \\
\text { Random } \\
10 \\
100 \\
\end{array}$ \\
\hline Application & $\begin{array}{l}\text { Data packet size } \\
\text { Broadcast packet size } \\
\text { Packet header size }\end{array}$ & $\begin{array}{l}100 \text { bytes } \\
25 \text { bytes } \\
25 \text { bytes }\end{array}$ \\
\hline Energy consumption model & $\begin{array}{c}E_{\text {elec }} \\
E_{\text {amp }}(\alpha, \beta)\end{array}$ & $\begin{array}{c}50 \mathrm{~nJ} / \mathrm{bit} \\
0.0013 \mathrm{pJ} / \mathrm{bit} / \mathrm{m}^{4}(0.003,0.001)\end{array}$ \\
\hline
\end{tabular}

3.3.1. Network Configuration Phase. The network configuration or setup phase is almost the same as in that in RARZ routing. The BS is responsible for dividing the whole sensing region into different cluster or zones by broadcasting different transmission power messages to the sensing region.

The main difference as compared to RARZ routing is that once the nodes receive the zone $i$ message from the BS, they will set their ID accordingly and inform the BS that they belong to zone $i$ by sending a join request message (join-req) back to the BS directly. This is an extra control message sent by all the nodes in the network which is an overhead. This process will be repeated at the start of each new round of communication. The complete working of the EEPSC is shown in Figure 2.

3.3.2. Data Communication Phase. Once the whole sensing region is divided into different clusters or zones, the data communication phase also called the steady phase begins. As the $\mathrm{BS}$ receives join request messages from all the sensor nodes in the sensing region, it randomly selects one temporary cluster head (TCH) in each zone and broadcast this information to all the clusters along with the time-division multiple-access (TDMA) schedule for each node. After that, the $\mathrm{TCH}$ will select one utmost energy level node as a $\mathrm{CH}$ and lowest energy level node as a TCH for the next round and inform all the nodes in the cluster. Afterward, all the nodes will send their sensed information to the designated $\mathrm{CH}$, and then the $\mathrm{CH}$ will send the accumulated information directly to the BS.

In this protocol, some major issues are identified. Firstly, all the nodes are directly reachable to each other. If all the nodes are directly reachable to each other, then there is no need for routing. Secondly, a lot of control information is needed for the selection of the $\mathrm{CH}$ and $\mathrm{TCH}$ before the start of each round of communication which is an overhead. The authors also claim that direct communication is energy efficient as compared to multihop routing which is not a valid point because we cannot 


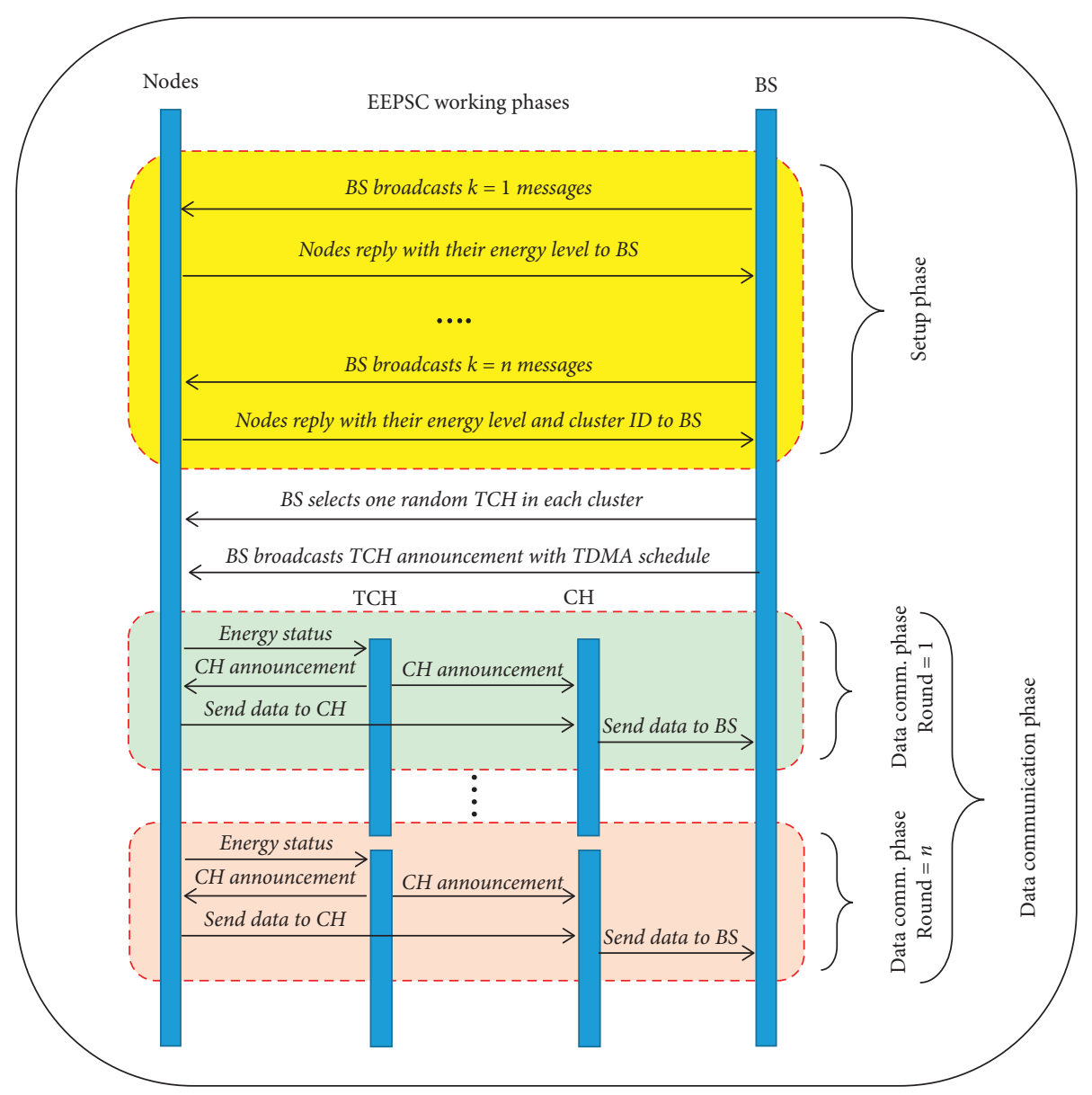

FIGURE 2: Working phases of the EEPSC.

compare the sensor node specification with the BS as the BS is more powerful and can cover the whole sensing region. And thirdly, if the network diameter is very large, then the nodes located at the edge of the network will require more transmission power to reach the BS and will deplete their energy very quickly. This greatly affects the overall network life. The assumptions made in the EEPSC are not valid as per the general prospects of the sensor networks. If all the nodes in the network are directly reachable to the BS, then because of the scalable environment, more collisions can occur in the network, which ultimately ends up in greater packet loss.

3.3.3. Energy Dissipation Model. The same energy model is used in simulation as used in the RARZ [2], EEHRT [9], EEPSC [7], and LEACH [11]. This is also called the firstorder radio model as developed and tested in [8]. According to this model, whenever a node sends or receives an $n$ bit message over distance $x$, energy will be consumed. For sending a message, the following model is utilized:

$$
\begin{aligned}
& E_{\mathrm{Tx}}(n, x)=E_{\mathrm{Tx}-\mathrm{elec}}(n)+E_{\mathrm{Tx}-\mathrm{amp}}(n, x), \\
& E_{\mathrm{Tx}}(n, x)=E_{\text {elec }} \times n+E_{\mathrm{amp}} \times n \times x^{2} .
\end{aligned}
$$

For receiving a message, the following model is utilized:

$$
\begin{aligned}
& E_{\mathrm{Rx}}(n)=E_{\mathrm{Rx}-\mathrm{elec}}(x), \\
& E_{\mathrm{Rx}}(n)=E_{\text {elec }} \times x .
\end{aligned}
$$

In (2), $E_{\mathrm{Tx}}(n, x)$ is the energy required to transmit an $n$ bit message over a distance of $x$ meters and $E_{\text {amp }}$ is the energy used for the amplifier to realize an acceptable signal-to-noise ratio (SNR). In (3), $E_{\mathrm{Rx}}(n)$ is the energy required to receive an $n$ bit message and $E_{\text {elec }}$ is the energy for transceiver circuitry.

3.4. Low-Energy Adaptive Clustering Hierarchy (LEACH). A plethora of work presented on routing stuff is available in the digital library. Among all the routing protocols developed till now, people have contributed a lot to cluster-based routing. LEACH [11] is based on distributed $\mathrm{CH}$ selection and cluster formation, where sensor nodes elect themselves as $\mathrm{CH}$ nodes with some probability based on remaining energy and location information. A centralized approach is also developed to form the clusters through the BS. LEACH-C is based on LEACH and uses a centralized approach for cluster formation and $\mathrm{CH}$ selection. Direct communication is formulated between $\mathrm{CH}$ and the BS. LEACH works in different phases or rounds. Each round starts with a configuration phase also called the network 


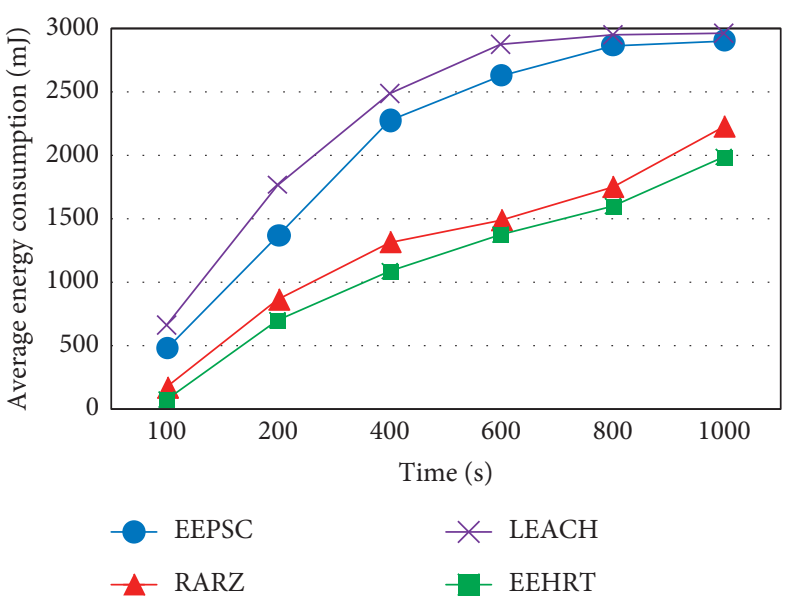

(a)

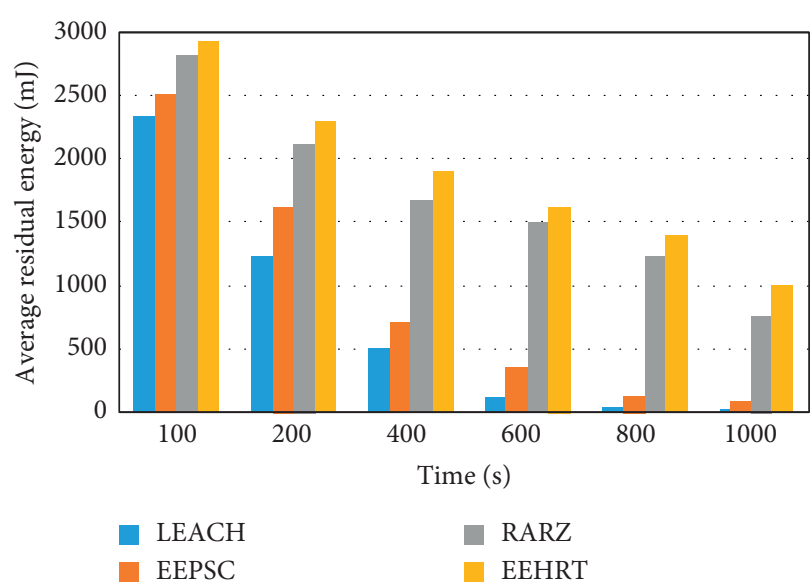

(b)

Figure 3: (a) Average energy consumption (b) and average residual energy in the system over time.

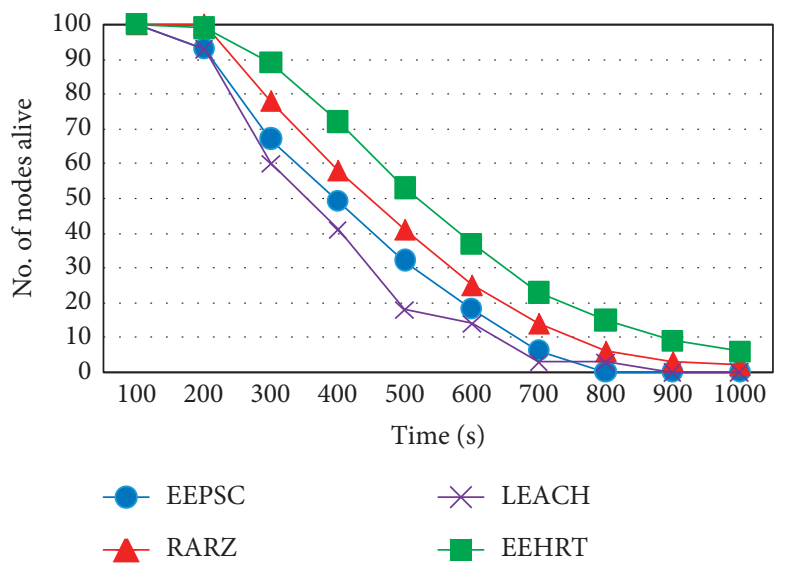

(a)

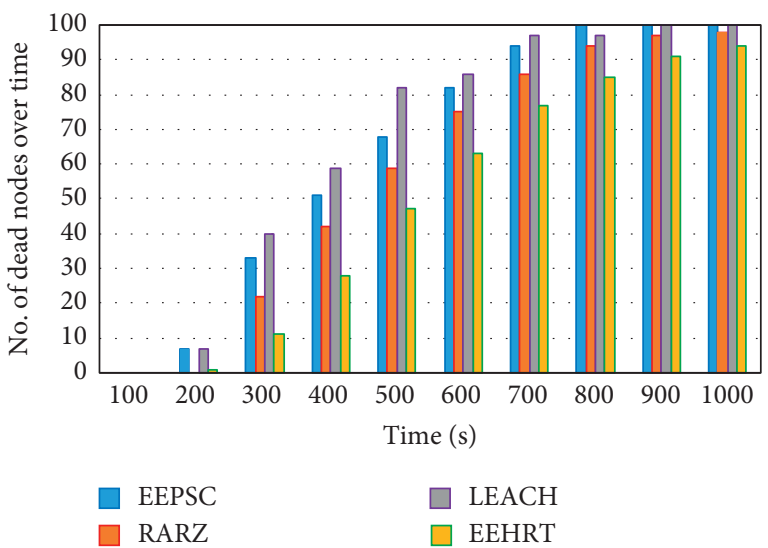

(b)

Figure 4: (a) Number of nodes alive (network lifetime) and (b) number of dead nodes over time.

setup phase followed by a data communication phase. Once all the nodes associate themselves with their designated cluster, they will send their data in their allotted time slot to the $\mathrm{CH}$, and then the $\mathrm{CH}$ will send the whole cluster data after making some necessary aggregation to the BS.

\section{Simulation and Result Discussion}

We compare the zone-based routing (ZBR) with hierarchical routing (HR) based on following metrics used in the simulation. Simulation is done in OMNeT++, using an INET framework [25].

(i) Average energy consumption and remaining energy in the system over time

(ii) The number of nodes alive and dead over time (network lifetime)

(iii) Messages successfully received at the BS over time, i.e., network throughput

We have used the same parameters as those used in the RARZ, EEHRT, LEACH, and EEPSC to assess the performance of all the protocols. The parameters of the simulation setup are listed in Table 1.

In Figures 3(a) and 3(b), the average energy consumption and remaining energy in the system over time are shown for all the protocols. The EEHRT and RARZ perform better as compared to the EEPSC and LEACH because in the RARZ and EEHRT, no extra control information is needed to set up the routing path and $\mathrm{CH}$ selection in the network. On the contrary, a lot of control packets are exchanged abundantly at the start of each round of communication in the EEPSC and LEACH which consumes most of the energy in the network. Various control packets are exchanged among the nodes to find the appropriate $\mathrm{CH}$, but the RARZ and EEHRT eliminated this overhead by selecting the next hop node without considering any control information that greatly affects the overall network lifetime.

Figures 4(a) and 4(b) show the number of nodes alive and dead over time, respectively. The EEHRT and RARZ outperform the EEPSC and LEACH in terms of a network lifetime because in the EEHRT and RARZ, the energy consumption is evenly distributed and the most energy level 


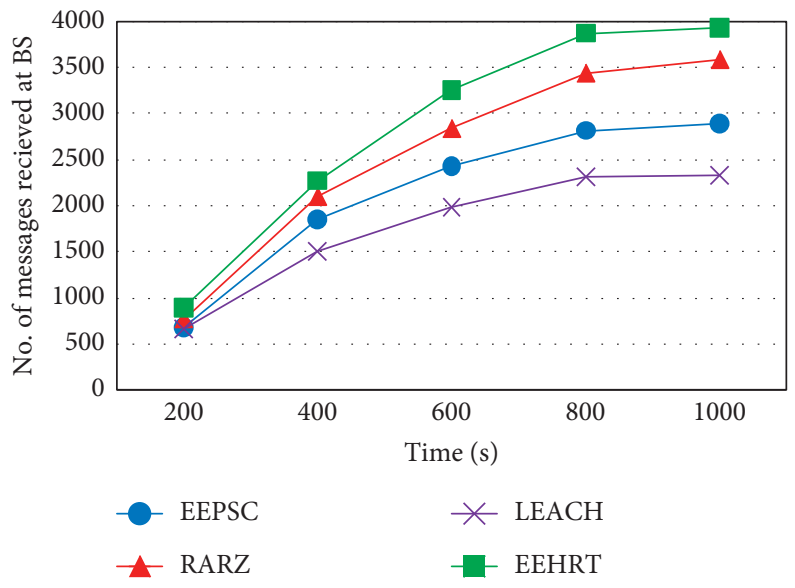

FIgURE 5: Number of data messages received at the BS over time.

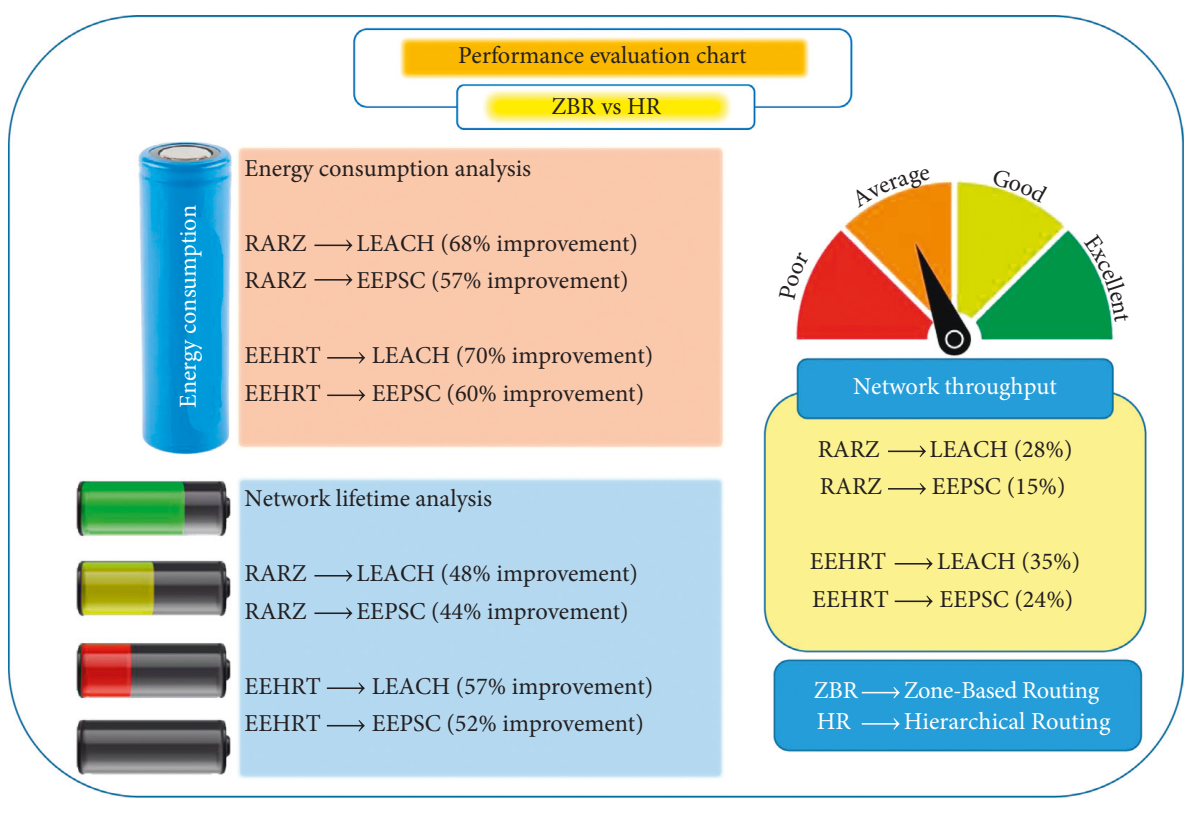

Figure 6: Performance analysis chart.

nodes are always engaged for the routing purpose. As shown in Figure 4, in the EEPSC and LEACH, all the nodes are alive for 100 seconds before the first node completely drains its energy, while in EEHRT and RARZ routing, even within $200 \mathrm{~s}$, none of the nodes in the network dies, which is better than the EEPSC.

The total number of messages successfully received at the BS is shown in Figure 5. It is clearly shown that the number of messages received at the BS is more in the EEHRT and RARZ as compared to that in the EEPSC and LEACH because whenever a node has data to send directly, it broadcasts its data in zone-based routing without exchanging any information before the start of each round of communication. If we thoroughly examine the routing procedure of the EEPSC and LEACH protocol, it is clearly seen that, before the actual data transmission, a lot of control information is exchanged for cluster setup and routing, but in EEHRT and RARZ routing, there is no such information needed for routing the data to the BS. The routing is totally blind, and routing is done on the fly without having any topological information stored in the network.

The performance improvement chart shows the comparative analysis of ZBR with HR in terms of energy efficiency, network throughput, and network lifetime. The general findings are presented in \% improvements. The detailed comparative analysis findings of ZBR against $\mathrm{HR}$ are presented in Figure 6.

\section{Conclusion}

In this paper, we have compared the performance of two different categories of routing protocols, i.e., zone-based routing $(\mathrm{ZBR})$ and hierarchical routing $(\mathrm{HR})$, in wireless sensor networks. The simulation shows that ZBR outperforms HR in terms of overall network lifetime, throughput, and energy efficiency. The main finding is that extra control information greatly affects the overall network lifetime and routing process. In ZBR (EEHRT and RARZ), it 
is shown that no extra control information is needed and exchanged among the nodes or with the BS while selecting the next hop node. ZBR does location-based routing without making any assumption of location services like GPS. It also improves the routing by giving more priority to those nodes which are located immediately to the next zone which greatly reduces the number of hops to the BS. Hence, it is concluded that ZBR outperforms HR in the sensor networks in terms of energy efficiency, network throughput, and overall network lifetime.

\section{Data Availability}

No data were used to support this study. We have conducted the simulations to evaluate the performance of zone-based routing with hierarchical routing. However, any query about the research conducted in this paper is highly appreciated and can be asked directly to the corresponding author through email (rabnawaz@mail.ustc.edu.cn).

\section{Conflicts of Interest}

The authors declare no conflicts of interest.

\section{Acknowledgments}

This work was financially supported by the National Natural Science Foundation of China (Grant No. 61631018). We are thankful to School of Information Science and technology (SIST), USTC, Hefei, China, and COMSATS University, Islamabad (Abbottabad Campus), which fully supported us by providing all key resources during the implementation and all later phases of this project.

\section{References}

[1] B. Bhushan and G. Sahoo, "Routing protocols in wireless sensor networks," in Computational Intelligence in Sensor Networks, pp. 215-248, Springer, Berlin, Germany, 2019.

[2] R. Jadoon, W. Zhou, W. Jadoon, and I. Ahmed Khan, "RARZ: ring-zone based routing protocol for wireless sensor networks," Applied Sciences, vol. 8, no. 7, p. 1023, 2018.

[3] M. V. Kumaramangalam, K. Adiyapatham, and C. Kandasamy, "Zone-based routing protocol for wireless sensor networks," International Scholarly Research Notices, vol. 2014, Article ID 798934, 9 pages, 2014.

[4] R. Nawaz, S. A. Hussain, S. A. Abid, and J. Shafi, "Beaconless multihop routing protocol for wireless sensor networks," in Proceedings of the 2011 IEEE 3rd International Conference on Communication Software and Networks, IEEE, Xi'an, China, May 2011.

[5] S. Lindsey and C. S. Raghavendra, "PEGASIS: power-efficient gathering in sensor information systems," in Proceedings of the IEEE Aerospace Conference, IEEE, Big Sky, MT, USA, March 2002.

[6] A. A. Awan, M. A. Khan, A. N. Malik et al., "Quality of service-based node relocation technique for mobile sensor networks," Wireless Communications and Mobile Computing, vol. 2019, Article ID 5043187, 13 pages, 2019.

[7] A. S. Zahmati and B. Abolhassani, "An energy-efficient protocol with static clustering for wireless sensor networks,"
International Journal of Electronics, Circuits and Systems, vol. 1, no. 2, pp. 135-138, 2007.

[8] M. Khari, "Wireless sensor networks: a technical survey," in Handbook of Research on Network Forensics and Analysis Techniques, pp. 1-18, IGI Global, Hershey, PA, USA, 2018.

[9] R. Nawaz Jadoon, W. Zhou, I. A. Khan, M. A. Khan, and W. Jadoon, "EEHRT: energy efficient technique for handling redundant traffic in zone-based routing for wireless sensor networks," Wireless Communications and Mobile Computing, vol. 2019, Article ID 7502140, 12 pages, 2019.

[10] J. N. Al-Karaki and A. E. Kamal, "Routing techniques in wireless sensor networks: a survey," IEEE Wireless Communications, vol. 11, no. 6, pp. 6-28, 2004.

[11] W. B. Heinzelman, A. P. Chandrakasan, and H. Balakrishnan, "An application-specific protocol architecture for wireless microsensor networks," IEEE Transactions on Wireless Communications, vol. 1, no. 4, pp. 660-670, 2002.

[12] W. R. Heinzelman, A. Chandrakasan, and H. Balakrishnan, "Energy-efficient communication protocol for wireless microsensor networks," in Proceedings of the 33rd Annual Hawaii International Conference on System Sciences, IEEE, Maui, Hawaii, January 2000.

[13] W. R. Heinzelman, A. Sinha, A. Wang, and A. P. Chandrakasan, "Energy-scalable algorithms and protocols for wireless microsensor networks," in Proceedings of the 2000 IEEE International Conference on Acoustics, Speech, and Signal Processing, IEEE, İstanbul, Turkey, June 2000.

[14] W. B. Heinzelman, Application-Specific Protocol Architectures for Wireless Networks, Massachusetts Institute of Technology, Cambridge, MA, USA, 2000.

[15] M. Hempel, H. Sharif, and P. Raviraj, "HEAR-SN: a new hierarchical energy-aware routing protocol for sensor networks," in Proceedings of the 38th Annual Hawaii International Conference on System Sciences, IEEE, Big Island, HI, USA, January 2005.

[16] D. Baker, A. Ephremides, and J. Flynn, "The design and simulation of a mobile radio network with distributed control," IEEE Journal on Selected Areas in Communications, vol. 2, no. 1, pp. 226-237, 1984.

[17] F. Bajaber and I. Awan, "Dynamic/static clustering protocol for wireless sensor network," in Proceedings of the Second UKSIM European Symposium on Computer Modeling and Simulation, IEEE, Athens, Greece, November 2008.

[18] M. Guerroumi and A.-S. K. Pathan, "Hybrid data dissemination protocol (HDDP) for wireless sensor networks," Wireless Networks, vol. 24, no. 5, pp. 1739-1754, 2018.

[19] N. Al-Humidi and G. V. Chowdhary, "Energy-aware approach for routing protocol by using centralized control clustering algorithm in wireless sensor networks," in Computing, Communication and Signal Processing, pp. 261-274, Springer, Berlin, Germany, 2019.

[20] R. Saini and M. Khari, "Defining malicious behavior of a node and its defensive techniques in ad hoc networks," International Journal of Smart Sensors and Ad Hoc Networks, vol. 1, no. 1, pp. 17-20, 2011.

[21] M. Khari, "Mobile ad hoc networks security attacks and secured routing protocols: a survey," in Proceedings of the International Conference on Computer Science and Information Technology, Springer, Hong Kong, China, December 2012.

[22] G. Shrivastava, Handbook of Research on Network Forensics and Analysis Techniques, IGI Global, Hershey, PA, USA, 2018.

[23] A. Maheshwari and N. Chand, "A survey on wireless sensor networks coverage problems," in Proceedings of 2nd 
International Conference on Communication, Computing and Networking, Springer, Chandigarh, India, March 2018.

[24] A. E. Khandani, "Cooperative routing in wireless networks," in Advances in Pervasive Computing and Networking, pp. 97-117, Springer, Berlin, Germany, 2005.

[25] A. Varga, "Discrete event simulation system," in Proceedings of the European Simulation Multiconference, Prague, Czech Republic, June 2001. 


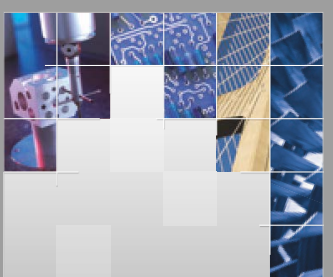

\section{Enfincering}
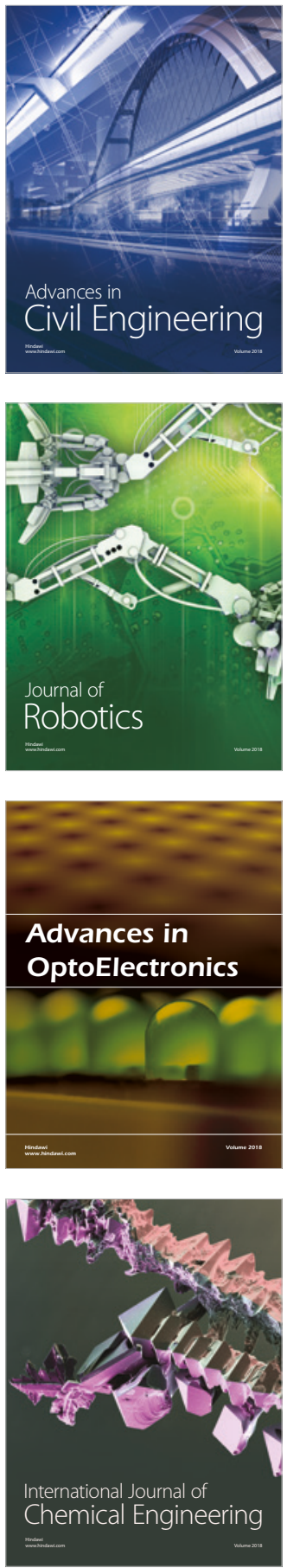

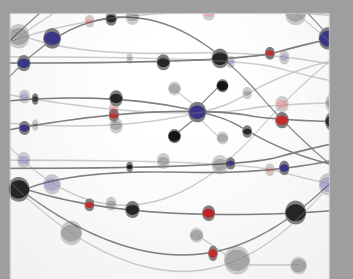

\section{Rotating \\ Machinery}

The Scientific World Journal

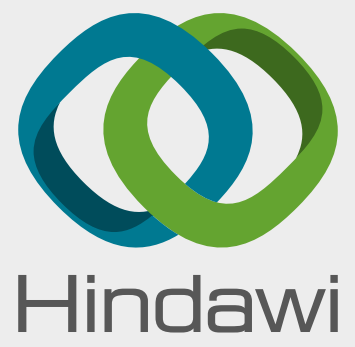

Submit your manuscripts at

www.hindawi.com
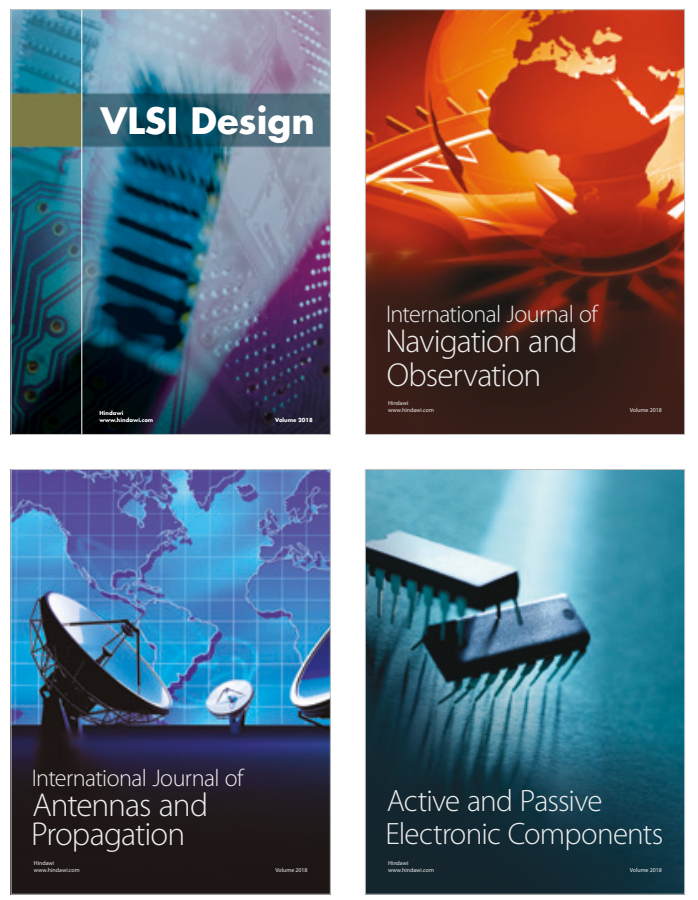
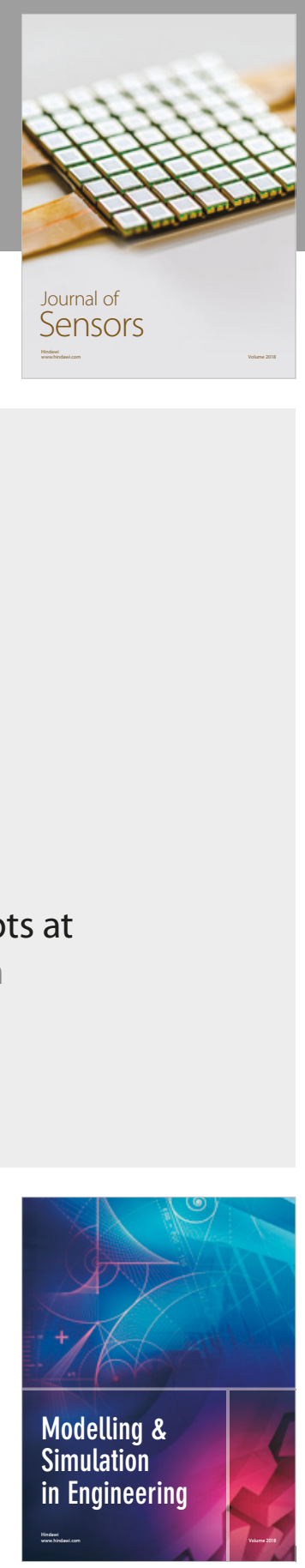

\section{Advances \\ Multimedia}
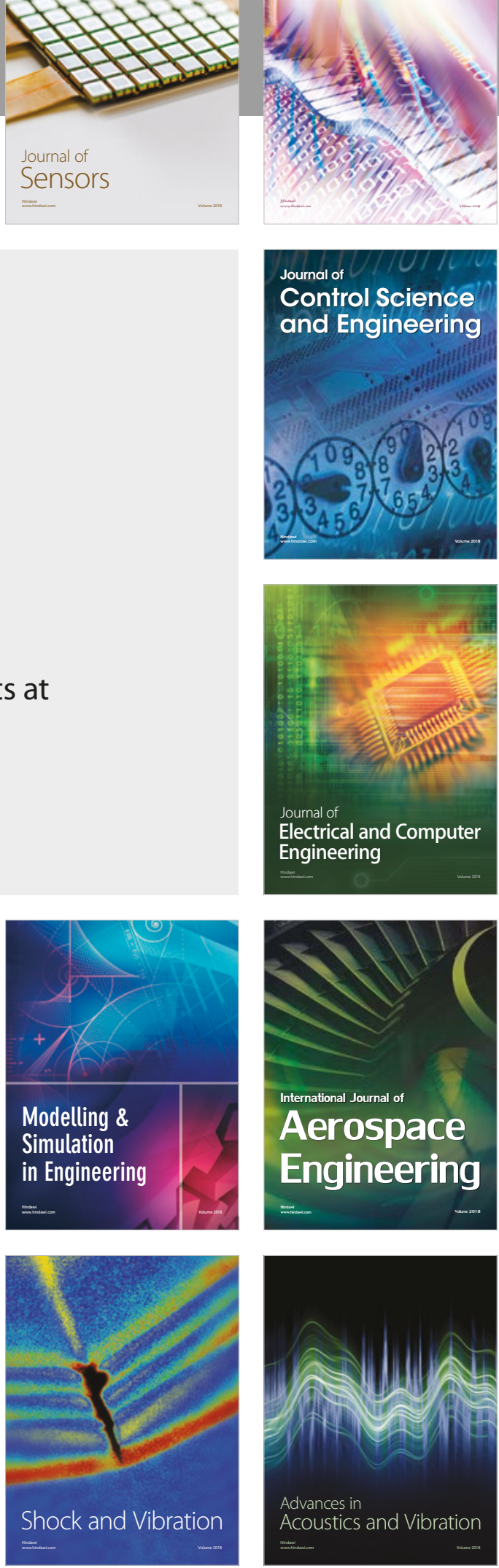